\title{
On direct and inverse spectral problems for sloshing of a two-layer fluid in an open container
}

\author{
N. G. Kuznetsov \\ Laboratory for Mathematical Modelling of Wave Phenomena, \\ Institute for Problems in Mechanical Engineering, Russian Academy of Sciences, \\ V. O., Bol'shoy pr. 61, St. Petersburg, 199178, Russian Federation \\ nikolay.g.kuznetsov@gmail.com
}

\begin{abstract}
We study the direct and inverse eigenvalue problems for a pair of harmonic functions with a spectral parameter in boundary and coupling conditions. The direct problem is relevant to sloshing frequencies of free oscillations of a two-layer fluid in a container. The upper fluid occupies a layer bounded above by a free surface and below by a layer of fluid of greater density. Both fluids are assumed to be inviscid, incompressible, and heavy, whereas the free surface and the interface between fluids are considered bounded.
\end{abstract}

Keywords: Laplace equation, sloshing problem, two-layer fluid, eigenvalue, eigenfunction, inverse spectral problem.

Received: 19 August 2016

Revised: 5 September 2016

In memory of B. S. Pavlov (1936-2016)

\section{Introduction}

Linear water wave theory is a widely used approach for describing the behavior of surface waves in the presence of rigid boundaries. In particular, this theory is a common tool for determining sloshing frequencies and modes in containers occupied by a homogeneous fluid, that is, having constant density. The corresponding boundary spectral problem, usually referred to as the sloshing problem, has been the subject of a great number of studies over more than two centuries (a historical review can be found, for example, in [1]). In the comprehensive book [2], an advanced technique based on spectral theory of operators in a Hilbert space was presented for studying this problem.

In the framework of the mathematical theory of linear water waves, substantial work has been done in the past two decades for understanding the difference between the results valid for homogeneous and two-layer fluids (in the latter case the upper fluid occupies a layer bounded above by a free surface and below by a layer of fluid whose density is greater than that in the upper one). These results concern wave/structure interactions and trapping of waves by immersed bodies (see, for example, [3-5] and references cited therein), but much less is known about the difference between sloshing in containers occupied by homogeneous and two-layer fluids. To the author's knowledge, there is only one related paper [6] with rigorous results for multilayered fluids, but it deals only with the spectral asymptotics in a closed container. Thus, the first aim of the present paper is to fill in this gap at least partially.

Another aim is to consider the so-called inverse sloshing problem; that is, the problem of recovering some physical parameters from known spectral data. The parameters to be recovered are the depth of the interface between the two layers and the density ratio that characterizes stratification. It is demonstrated that for determining these two characteristics for fluids occupying a vertical-walled container with a horizontal bottom, one has to measure not only the two smallest sloshing eigenfrequencies, which must satisfy certain inequalities, but also to analyze the corresponding free surface elevations.

\subsection{Statement of the direct problem}

Let two immiscible, inviscid, incompressible, heavy fluids occupy an open container whose walls and bottom are rigid surfaces. We choose rectangular Cartesian coordinates $\left(x_{1}, x_{2}, y\right)$ so that their origin lies in the mean free surface of the upper fluid and the $y$-axis is directed upwards. Then, the whole fluid domain $W$ is a subdomain of the lower half-space $\left\{-\infty<x_{1}, x_{2}<+\infty, y<0\right\}$. The boundary $\partial W$ is assumed to be piece-wise smooth and such that every two adjacent smooth pieces of $\partial W$ are not tangent along their common edge. We also suppose that each horizontal cross-section of $W$ is a bounded two-dimensional domain; that is, a connected, open set in the corresponding plane. (The latter assumption is made for the sake of simplicity because it excludes the possibility of two or more interfaces between fluids at different levels.) The free surface $F$ bounding above the 
upper fluid of density $\rho_{1}>0$ is the non-empty interior of $\partial W \cap\{y=0\}$. The interface $I=W \cap\{y=-h\}$, where $0<h<\max \left\{|y|:\left(x_{1}, x_{2}, y\right) \in \partial W\right\}$, separates the upper fluid from the lower one of density $\rho_{2}>\rho_{1}$. We denote by $W_{1}$ and $W_{2}$ the domains $W \cap\{y>-h\}$ and $W \cap\{y<-h\}$ respectively; they are occupied by the upper and lower fluids respectively. The surface tension is neglected and we suppose the fluid motion to be irrotational and of small amplitude. Therefore, the boundary conditions on $F$ and $I$ may be linearized. With a time-harmonic factor, say cos $\omega t$, removed, the velocity potentials $u^{(1)}\left(x_{1}, x_{2}, y\right)$ and $u^{(2)}\left(x_{1}, x_{2}, y\right)$ (they may be taken to be real functions) for the flow in $W_{1}$ and $W_{2}$ respectively must satisfy the following coupled boundary value problem:

$$
\begin{aligned}
& u_{x_{1} x_{1}}^{(j)}+u_{x_{2} x_{2}}^{(j)}+u_{y y}^{(j)}=0 \quad \text { in } W_{j}, \quad j=1,2, \\
& u_{y}^{(1)}=\nu u^{(1)} \text { on } F, \\
& \rho\left(u_{y}^{(2)}-\nu u^{(2)}\right)=u_{y}^{(1)}-\nu u^{(1)} \quad \text { on } I, \\
& u_{y}^{(2)}=u_{y}^{(1)} \text { on } I, \\
& \partial u^{(j)} / \partial n=0 \quad \text { on } B_{j} \quad j=1,2 .
\end{aligned}
$$

Here, $\rho=\rho_{2} / \rho_{1}>1$ is the non-dimensional measure of stratification, the spectral parameter $\nu$ is equal to $\omega^{2} / g$, where $\omega$ is the radian frequency of the water oscillations and $g$ is the acceleration due to gravity; $B_{j}=\partial W_{j} \backslash(\bar{F} \cup \bar{I})$ is the rigid boundary of $W_{j}$. By combining (3) and (4), we get another form of the spectral coupling condition (3):

$$
(\rho-1) u_{y}^{(2)}=\nu\left(\rho u^{(2)}-u^{(1)}\right) \quad \text { on } I \text {. }
$$

We also suppose that the orthogonality conditions

$$
\int_{F} u^{(1)} \mathrm{d} x=0 \quad \text { and } \int_{I}\left(\rho u^{(2)}-u^{(1)}\right) \mathrm{d} x=0, \quad \mathrm{~d} x=\mathrm{d} x_{1} \mathrm{~d} x_{2},
$$

hold, thus excluding the zero eigenvalue of (1)-(5).

When $\rho=1$, conditions (3) and (4) mean that the functions $u^{(1)}$ and $u^{(2)}$ are harmonic continuations of each other across the interface $I$. Then, problem (1)-(5) complemented by the first orthogonality condition (7) (the second condition (7) is trivial), becomes the usual sloshing problem for a homogeneous fluid. It is well-known since the 1950s that the latter problem has a positive discrete spectrum. This means that there exists a sequence of positive eigenvalues $\left\{\nu_{n}^{W}\right\}_{1}^{\infty}$ of finite multiplicity (the superscript $W$ is used here and below for distinguishing the sloshing eigenvalues that correspond to the case, when a homogeneous fluid occupies the whole domain $W$, from those corresponding to a two-layer fluid which will be denoted simply by $\nu_{n}$ ). In this sequence the eigenvalues are written in increasing order and repeated according to their multiplicity; moreover, $\nu_{n}^{W} \rightarrow \infty$ as $n \rightarrow \infty$. The corresponding eigenfunctions $\left\{u_{n}\right\}_{1}^{\infty} \subset H^{1}(W)$ form a complete system in an appropriate Hilbert space. These results can be found in many sources, for example, in the book [2].

\section{Variational principle}

Let $W$ be bounded. It is well known that the sloshing problem in $W$ for homogeneous fluid can be cast into the form of a variational problem and the corresponding Rayleigh quotient is as follows:

$$
R_{W}(u)=\frac{\int_{W}|\nabla u|^{2} \mathrm{~d} x y}{\int_{F} u^{2} \mathrm{~d} x} .
$$

In order to obtain the fundamental eigenvalue $\nu_{1}^{W}$ one has to minimize $R_{W}(u)$ over the subspace of the Sobolev space $H^{1}(W)$ consisting of functions that satisfy the first orthogonality condition (7). To find $\nu_{n}^{W}$ for $n>1$, one has to minimize (8) over the subspace of $H^{1}(W)$ such that each of its element $u$ satisfies the first condition (7) along with the following equalities $\int_{F} u u_{j} \mathrm{~d} x=0$, where $u_{j}$ is either of the eigenfunctions $u_{1}, \ldots, u_{n-1}$ corresponding to the eigenvalues $\nu_{1}^{W}, \ldots, \nu_{n-1}^{W}$.

In the case of a two-layer fluid, we assume that the usual embedding theorems hold for both subdomains $W_{j}$, $j=1,2$ (the theorem about traces on smooth pieces of the boundary for elements of $H^{1}$ included). This imposes some restrictions on $\partial W$, in particular, on the character of the intersections of $F$ and $I$ with $\partial W \cap\{y<0\}$. Then 
using (6), it is easy to verify that the Rayleigh quotient for the two-layer sloshing problem has the following form:

$$
R\left(u^{(1)}, u^{(2)}\right)=\frac{\int_{W_{1}}\left|\nabla u^{(1)}\right|^{2} \mathrm{~d} x \mathrm{~d} y+\rho \int_{W_{2}}\left|\nabla u^{(2)}\right|^{2} \mathrm{~d} x \mathrm{~d} y}{\int_{F}\left[u^{(1)}\right]^{2} \mathrm{~d} x+(\rho-1)^{-1} \int_{I}\left[\rho u^{(2)}-u^{(1)}\right]^{2} \mathrm{~d} x} .
$$

To determine the fundamental sloshing eigenvalue $\nu_{1}$ one has to minimize $R\left(u^{(1)}, u^{(2)}\right)$ over the subspace of $H^{1}\left(W_{1}\right) \oplus H^{1}\left(W_{2}\right)$ defined by both orthogonality conditions (7). In order to find $\nu_{n}$ for $n>1$, one has to minimize (9) over the subspace of $H^{1}\left(W_{1}\right) \oplus H^{1}\left(W_{2}\right)$ such that every element $\left(u^{(1)}, u^{(2)}\right)$ of this subspace satisfies the equalities:

$$
\int_{F} u^{(1)} u_{j}^{(1)} \mathrm{d} x=0 \text { and } \int_{I}\left[\rho u^{(2)}-u^{(1)}\right]\left[\rho u_{j}^{(2)}-u_{j}^{(1)}\right] \mathrm{d} x=0, \quad j=1, \ldots, n-1,
$$

along with both conditions (7). Here, $\left(u_{j}^{(1)}, u_{j}^{(2)}\right)$ is either of the eigensolutions corresponding to $\nu_{1}, \ldots, \nu_{n-1}$.

Now we are in a position to prove the following assertion.

Proposition 1. Let $\nu_{1}^{W}$ and $\nu_{1}$ be the fundamental eigenvalues of the sloshing problem in the bounded domain $W$ for homogeneous and two-layer fluids respectively. Then the inequality $\nu_{1}<\nu_{1}^{W}$ holds.

The restriction that $W$ is bounded is essential as the example considered in Proposition 4 below demonstrates. Proof. If $u_{1}$ is an eigenfunction corresponding to $\nu_{1}^{W}$, then

$$
\nu_{1}^{W}=\frac{\int_{W}\left|\nabla u_{1}\right|^{2} \mathrm{~d} x \mathrm{~d} y}{\int_{F} u_{1}^{2} \mathrm{~d} x} .
$$

Let $u^{(1)}$ and $u^{(2)}$ be equal to the restrictions of $\rho u_{1}$ and $u_{1}$ to $W_{1}$ and $W_{2}$, respectively. Then the pair $\left(u^{(1)}, u^{(2)}\right)$ is an admissible element for the Rayleigh quotient (9). Substituting it into (9), we obtain that:

$$
R\left(\rho u_{1}, u_{1}\right)=\frac{\int_{W_{1}}\left|\nabla u_{1}\right|^{2} \mathrm{~d} x \mathrm{~d} y+\rho^{-1} \int_{W_{2}}\left|\nabla u_{1}\right|^{2} \mathrm{~d} x \mathrm{~d} y}{\int_{F} u_{1}^{2} \mathrm{~d} x} .
$$

Comparing this equality with the previous one and taking into account that $\rho>1$, one finds that $R\left(\rho u_{1}, u_{1}\right)<\nu_{1}^{W}$. Since $\nu_{1}$ is the minimum of (9), we conclude that $\nu_{1}<\nu_{1}^{W}$.

\section{Containers with vertical walls and horizontal bottoms}

Let us consider the fluid domain $W=\left\{x=\left(x_{1}, x_{2}\right) \in D, y \in(-d, 0)\right\}$, where $D$ is a piece-wise smooth two-dimensional domain (the container's horizontal cross-section) and $d \in(0, \infty]$ is the container's constant depth. Thus, the container's side wall $\partial D \times(-d, 0)$ is vertical, the bottom $\{x \in D, y=-d\}$ is horizontal, whereas the free surface and the interface are $F=\{x \in D, y=0\}$ and $I=\{x \in D, y=-h\}$ respectively, $0<h<d$.

For a homogeneous fluid occupying such a container, the sloshing problem is equivalent to the free membrane problem. Indeed, putting

$$
u(x, y)=v(x) \cosh k(y+d) \quad\left(u(x, y)=v(x) \mathrm{e}^{k y} \text { when } d=\infty\right),
$$

one reduces problem (1)-(5) with $\rho=1$, complemented by the first orthogonality condition (7) to the following spectral problem:

$$
\nabla_{x}^{2} v+k^{2} v=0 \text { in } D, \quad \partial v / \partial n_{x}=0 \quad \text { on } \partial D, \quad \int_{D} v \mathrm{~d} x=0,
$$

where $\nabla_{x}=\left(\partial / \partial x_{1}, \partial / \partial x_{2}\right)$ and $n_{x}$ is a unit normal to $\partial D$ in $\mathbb{R}^{2}$. It is clear that $\nu^{W}$ is an eigenvalue of the former problem if and only if $k^{2}$ is an eigenvalue of (10) and

$$
\nu^{W}=k \tanh k d \quad \text { when } d<\infty \quad\left(\nu^{W}=k \quad \text { when } d=\infty\right), \quad k>0 .
$$

It is well-known that problem (10) has a sequence of positive eigenvalues $\left\{k_{n}^{2}\right\}_{1}^{\infty}$ written in increasing order and repeated according to their finite multiplicity, and such that $k_{n}^{2} \rightarrow \infty$ as $n \rightarrow \infty$. The corresponding eigenfunctions form a complete system in $H^{1}(D)$. 

Putting

Let us describe the same reduction procedure in the case when $W$ is occupied by a two-layer fluid and $d<\infty$.

$$
\begin{aligned}
& u^{(1)}(x, y)=v(x)[A \cosh k(y+h)+B \sinh k(y+h)], \\
& u^{(2)}(x, y)=v(x) C \cosh k(y+d),
\end{aligned}
$$

where $A, B$ and $C$ are constants, one reduces problem (1)-(5) and (7), $\rho>1$, to problem (10) combined with the following quadratic equation:

$$
\begin{aligned}
& \nu^{2} \cosh k d-\nu k[\sinh k d+(\rho-1) \cosh k h \sinh k(d-h)] \\
& \quad+k^{2}(\rho-1) \sinh k h \sinh k(d-h)=0, \quad k>0 .
\end{aligned}
$$

Thus $\nu$ is an eigenvalue of the former problem if and only if $\nu$ satisfies (14), where $k^{2}$ is an eigenvalue of (10).

Indeed, the quadratic polynomial in $\nu$ on the left-hand side of (14) is the determinant of the following linear algebraic system for $A, B$ and $C$ :

$$
\begin{array}{r}
A=C\left[\cosh k(d-h)-\nu^{-1}(\rho-1) k \sinh k(d-h)\right], \quad B=C \sinh k(d-h), \\
A(k \sinh k h-\nu \cosh k h)+C \sinh k(d-h)(k \cosh k h-\nu \sinh k h)=0 .
\end{array}
$$

The latter arises when one substitutes expressions (12) and (13) into the boundary condition (2) and the coupling conditions (3) and (4). This homogeneous system defines eigensolutions of the sloshing problem provided there exists a non-trivial solution, and so the determinant must vanish which is expressed by (14).

Let us show that the roots $\nu^{(+)}$and $\nu^{(-)}$of (14) are real in which case

$$
\nu^{( \pm)}=k \frac{b \pm \sqrt{\mathcal{D}}}{2 \cosh k d}>0,
$$

where the inequality is a consequence of the formulae:

$$
\begin{aligned}
& b=\sinh k d+(\rho-1) \cosh k h \sinh k(d-h), \\
& \mathcal{D}=b^{2}-4(\rho-1) \cosh k d \sinh k h \sinh k(d-h) .
\end{aligned}
$$

Since $\mathcal{D}$ is a quadratic polynomial of $\rho-1$, it is a simple application of calculus to demonstrate that it attains the minimum at

and after some algebra one finds that this minimum is equal to

$$
\rho-1=\frac{2 \cosh k d \sinh k h-\sinh k d \cosh k h}{\cosh ^{2} k h \sinh k(d-h)},
$$

$$
\frac{4 \cosh k d \sinh k h \sinh k(d-h)}{\cosh ^{2} k h}>0,
$$

which proves the assertion. Thus we arrive at the following.

Proposition 2. If $W$ is a vertical cylinder with horizontal bottom, then the sloshing problem for a two-layer fluid occupying $W$ has two sequences of eigenvalues

$$
\left\{\nu_{n}^{(+)}\right\}_{1}^{\infty} \text { and }\left\{\nu_{n}^{(-)}\right\}_{1}^{\infty}
$$

defined by (17) with $k=k_{n}>0$, where $k_{n}^{2}$ is an eigenvalue of problem (10).

The same eigensolution $\left(u^{(1)}, u^{(2)}\right)$ corresponds to both $\nu_{n}^{(+)}$and $\nu_{n}^{(-)}$, where $u^{(1)}$ and $u^{(2)}$ (sloshing modes in $W_{1}$ and $W_{2}$ respectively) are defined by formulae (12) and (13) with $v$ belonging to the set of eigenfunctions of problem (10) that correspond to $k_{n}^{2}$; furthermore, $C$ is an arbitrary non-zero real constant, whereas $A$ and $B$ depend on $C$ through (15).

Next, we analyze the behavior of $\nu_{n}^{( \pm)}$as a function of $\rho$.

Proposition 3. For every $n=1,2, \ldots$ the functions $\nu_{n}^{(-)}$and $\nu_{n}^{(+)}$are monotonically increasing as $\rho$ goes from 1 to infinity. Their ranges are:

$$
\left(0, k_{n} \tanh k_{n} h\right) \quad \text { and } \quad\left(k_{n} \tanh k_{n} d, \infty\right)
$$

respectively. 
Proof. In order to prove the proposition, it is sufficient to show that:

$$
\begin{aligned}
& \frac{\partial(b \pm \sqrt{\mathcal{D}})}{\partial \rho}=\sinh k(d-h)\left\{\cosh k h \pm \mathcal{D}^{-1 / 2}[\cosh k h \sinh k d\right. \\
& \left.\left.\quad+(\rho-1) \cosh ^{2} k h \sinh k(d-h)-2 \cosh k d \sinh k h\right]\right\}>0 .
\end{aligned}
$$

Since

$$
\left.\frac{\partial(b+\sqrt{\mathcal{D}})}{\partial \rho}\right|_{\rho=1}=\frac{2 \sinh ^{2} k(d-h)}{\sinh k d}>0 \quad \text { and }\left.\quad \frac{\partial(b-\sqrt{\mathcal{D}})}{\partial \rho}\right|_{\rho \rightarrow \infty}=0,
$$

inequality (20) is a consequence of the following one:

$$
\pm \frac{\partial^{2}(b \pm \sqrt{\mathcal{D}})}{\partial \rho^{2}}=\frac{4 \cosh k d \sinh k h \sinh ^{3} k(d-h)}{\mathcal{D}^{3 / 2}}>0 \quad \text { for all } \rho>1 \text {. }
$$

The second assertion immediately follows from the first one and formulae (17)-(19).

Combining Proposition 3 and formula (11), we arrive at the following assertion.

Corollary 1. The inequalities $\nu_{n}^{(-)}<\nu_{n}^{W}<\nu_{n}^{(+)}$hold for each $n=1,2, \ldots$ and every $\rho>1$.

Dividing (17) by $k$ and letting $k=k_{n}$ to infinity, it is straightforward to obtain the following.

Lemma 1. For every $\rho>1$, the asymptotic formula:

$$
\nu_{n}^{( \pm)} \sim \frac{\rho+1 \pm|\rho-3|}{4} k_{n} \quad \text { as } n \rightarrow \infty,
$$

holds with the exponentially small remainder term; here $k_{n}^{2}$ is an eigenvalue of (10).

In other words, there are three cases:

$$
\begin{aligned}
& \text { (i) if } \rho=3 \text {, then } \nu_{n}^{( \pm)} \sim k_{n} \text { as } n \rightarrow \infty \text {; } \\
& \text { (ii) if } \rho>3 \text {, then } \nu_{n}^{(-)} \sim k_{n} \text { and } \nu_{n}^{(+)} \sim(\rho-1) k_{n} / 2 \text { as } n \rightarrow \infty \text {; } \\
& \text { (iii) if } \rho \in(1,3) \text {, then } \nu_{n}^{(-)} \sim(\rho-1) k_{n} / 2 \text { and } \nu_{n}^{(+)} \sim k_{n} \text { as } n \rightarrow \infty \text {. }
\end{aligned}
$$

Combining these relations and the asymptotic formula $\nu_{n}^{W} \sim k_{n}$ as $n \rightarrow \infty$ (it is a consequence of formula (11) defining $\nu_{n}^{W}$ when a homogeneous fluid occupies $W$ ), we obtain the following.

Corollary 2. As $n \rightarrow \infty$, we have that $\nu_{n}^{(-)} \sim \nu_{n}^{W}$ when $\rho \geq 3$, whereas $\nu_{n}^{(+)} \sim \nu_{n}^{W}$ provided $\rho \in(1,3]$.

Another corollary of Lemma 1 concerns the distribution function $\mathcal{N}(\nu)$ for the spectrum of problem (1)-(5) and (7). This function is equal to the total number of eigenvalues $\nu_{n}$ that do not exceed $\nu$. An asymptotic formula for $\mathcal{N}(\nu)$ immediately follows from Lemma 1 and the asymptotic formula for the distribution of the spectrum for the Neumann Laplacian (see [7], Chapter 6).

Corollary 3. The distribution function $\mathcal{N}(\nu)$ of the spectrum for the sloshing of a two-layer fluid in a vertical cylinder of cross-section $D$ has the following asymptotics:

$$
\mathcal{N}(\nu) \sim\left[\frac{4}{(\rho-1)^{2}}+1\right] \frac{|D| \nu^{2}}{4 \pi} \text { as } \nu \rightarrow \infty
$$

Here, $|D|$ stands for the area of $D$.

It should be also mentioned that in [6] the asymptotics for $\mathcal{N}(\nu)$ was obtained for a multi-layer fluid occupying a bounded closed container.

It follows from Lemma 1 and Corollary 2 that the asymptotic formula for the distribution function of the spectrum $\left\{\nu_{n}^{W}\right\}_{1}^{\infty}$ is similar to the above one, but the first term in the square brackets must be deleted. Moreover, in the case of homogeneous fluid the same asymptotic formula (up to the remainder term) holds for arbitrarily shaped fluid domains (see [2], Section 3.3). Since the first term in the square brackets tends to infinity as $\rho \rightarrow 1$, the transition from the two-layer fluid to the homogeneous one in the asymptotic formula for $\mathcal{N}(\nu)$ is a singular limit in the sense described in [8]. A similar effect occurs for modes trapped by submerged bodies in two-layer and homogeneous fluids as was noted in [4].

In conclusion of this section, it should be noted that in the case of an infinitely deep vertical cylinder it is easy to verify that $\nu=k$ is an eigenvalue of the sloshing problem for a two-layer fluid if and only if $k^{2}$ is an eigenvalue of problem (10). Comparing this assertion with that at the beginning of this section, we obtain the following. 
Proposition 4. In an infinitely deep vertical-walled container, the sloshing problem for a two-layer fluid has the same set of eigenvalues and the same eigenfunctions of the form $v(x) \mathrm{e}^{k y}, k>0$, as the sloshing problem for a homogeneous fluid in the same container; here, $k^{2}$ is an eigenvalue and $v$ is the corresponding eigenfunction of problem (10).

\section{Inverse problem}

Let a given container $W$ be occupied by a two-layer fluid, but now we assume that the position of the interface between layers and the density of the lower layer are unknown. The density of the upper layer is known because one can measure it directly. The sequence of eigenvalues $\left\{\nu{ }_{n}^{W}\right\}_{1}^{\infty}$ corresponding to the homogeneous fluid is also known because it depends only on the domain $W$. The inverse problem we are going to consider is to recover the ratio of densities $\rho$ and the depth of the interface $h$ from measuring some sloshing frequencies on the free surface. Thus, we let the fundamental eigenvalue $\nu_{1}$ be known along with the second-largest one.

The formulated inverse problem is not always solvable. Indeed, according to Proposition 4, it has no solution when $W$ is an infinitely deep container with vertical walls. Moreover, the inverse problem is trivial for all domains when $\nu_{1}=\nu_{1}^{W}$. In this case, Proposition 1 implies that the fluid is homogeneous, that is, $\rho=1$ and $h=d$. Therefore, we restrict ourselves to the case of vertically-walled containers having a finite depth $d$ in what follows.

\subsection{Reduction to transcendental equations}

In view of what was said above, the inverse problem for $W=D \times(-d, 0)$ can be stated as follows. Find conditions that allow us to determine $\rho>1$ and $h \in(0, d)$ when the following two eigenvalues are known: the fundamental one $\nu_{1}$ and the smallest eigenvalue $\nu_{N}$ that is greater than $\nu_{1}$. Thus, $N$ is such that $k_{n}^{2}=k_{1}^{2}$ for all $n=1, \ldots, N-1$, which means that the fundamental eigenvalue $k_{1}^{2}$ of problem (10) is of multiplicity $N-1$ (of course, $\nu_{1}$ has the same multiplicity). For example, if $D$ is a disc, then the multiplicity of $k_{1}^{2}$ is two (see [9], Section 3.1), and so $\nu_{N}=\nu_{3}$ in this case.

According to formula (17), we have that $\nu_{1}=\nu_{1}^{(-)}$. Hence the first equation for $\rho$ and $h$ is as follows:

$$
b_{1}-\sqrt{\mathcal{D}_{1}}=\frac{2 \nu_{1}}{k_{1}} \cosh k_{1} d .
$$

Here, $b_{1}$ and $\mathcal{D}_{1}$ are given by formulae (18) and (19) respectively with $k=k_{1}$.

To write down the second equation for $\rho$ and $h$, we have the dilemma whether

$$
\nu_{N}=\nu_{N}^{(-)} \quad \text { or } \quad \nu_{N}=\nu_{1}^{(+)} ?
$$

Let us show that either of these options is possible. Indeed, Proposition 3 implies that $\nu_{N}=\nu_{N}^{(-)}$provided $\rho-1$ is sufficiently small. On the other hand, let us demonstrate that there exists a triple $(\rho, d, h)$ for which $\nu_{N}=\nu_{1}^{(+)}$. For this purpose we have to demonstrate that the inequality

$$
\nu_{N}^{(-)}=k_{N} \frac{b_{N}-\sqrt{\mathcal{D}_{N}}}{2 \cosh k_{N} d} \geq k_{1} \frac{b_{1}+\sqrt{\mathcal{D}_{1}}}{2 \cosh k_{1} d}=\nu_{1}^{(+)}
$$

holds for some $\rho, d$ and $h$. As above $b_{j}$ and $\mathcal{D}_{j}, j=1, N$, are given by formulae (18) and (19), respectively, with $k=k_{j}$.

Let $h=d / 2$, then we have:

$$
4 \nu_{j}^{( \pm)}=k_{j}\left\{(\rho+1) \tanh k_{j} d \pm\left[(\rho+1)^{2} \tanh ^{2} k_{j} d+8(\rho-1) \frac{1-\cosh k_{j} d}{\cosh k_{j} d}\right]^{1 / 2}\right\}
$$

and so

$$
4\left[\nu_{N}^{(-)}-\nu_{1}^{(+)}\right] \rightarrow k_{N}(\rho+1-|\rho-3|)-k_{1}(\rho+1+|\rho-3|) \quad \text { as } d \rightarrow \infty
$$

The limit is piecewise linear function of $\rho$, attains its maximum value $4\left(k_{N}-k_{1}\right)$ at $\rho=3$ and is positive for $\rho \in\left(1+2\left(k_{1} / k_{N}\right), 1+2\left(k_{N} / k_{1}\right)\right)$.

Summarizing, we arrive at the following.

Proposition 5. Let $k_{N}^{2}$ be the smallest eigenvalue of problem (10) other than $k_{1}^{2}$, and let $\nu_{N}^{(-)}$be the sloshing eigenvalue defined by (17)-(19) with $k=k_{N}$. Then $D)$;

(i) $\nu_{N}^{(-)}<\nu_{1}^{(+)}$when $\rho-1>0$ is sufficiently small (of course, its value depends on $d, h$ and the domain

(ii) $\nu_{N}^{(-)}>\nu_{1}^{(+)}$when $\rho \in\left(1+2\left(k_{1} / k_{N}\right), 1+2\left(k_{N} / k_{1}\right)\right), h=d / 2$ and $d$ is sufficiently large (of course, its value depends on $\rho$ and $D$ ). 
Obviously, assertion (ii) can be extended to values of $h$ that are sufficiently close to $d / 2$.

\subsection{Options for the second equation}

Let us develop a procedure for determining which of the two equalities (22) can be chosen to complement equation (21) in order to find $\rho$ and $h$. Our procedure is based on an analysis of the free surface elevations corresponding to the measured values $\nu_{1}$ and $\nu_{N}$. Indeed, when a two-layer fluid oscillates at the frequency defined by some $\nu_{j}$, the free surface elevation is proportional to the trace $u_{j}^{(1)}(x, 0)$ (see, for example, [10], Section 227).

According to formula (12), the trace $u_{1}^{(1)}(x, 0)$ is a linear combination of linearly independent eigenfunctions $v_{1}(x), \ldots, v_{N-1}(x)$ corresponding to the fundamental eigenvalue $k_{1}^{2}$ of problem (10); of course, its multiplicity is taken into account. By Proposition 2 the free surface elevation associated with $\nu_{1}^{(+)}$is also proportional to a linear combination of $v_{1}, \ldots, v_{N-1}$. Since these functions are known, one has to determine whether the measured free-surface elevation corresponding to $\nu_{N}$ can be represented in the form of such a combination and only in such a form. If this is the case, then $\nu_{N}=\nu_{1}^{(+)}<\nu_{N}^{(-)}$and the following equation:

$$
b_{1}+\sqrt{\mathcal{D}_{1}}=\frac{2 \nu_{N}}{k_{1}} \cosh k_{1} d
$$

forms the system for $\rho$ and $h$ together with (21).

Besides, it can occur that the measured free-surface elevation corresponding to $\nu_{N}$ is representable in two forms, one of which is a linear combination of $v_{1}, \ldots, v_{N-1}$, whereas the other one involves the function $v_{N}$ as well as other eigenfunctions corresponding to the eigenvalue $k_{N}^{2}$ of problem (10) along with $v_{1}, \ldots, v_{N-1}$. It is clear that this happens when $\nu_{N}=\nu_{1}^{(+)}=\nu_{N}^{(-)}$. Indeed, if all coefficients at the eigenfunctions of $k_{N}^{2}$ vanish, then the profile is represented by $v_{1}, \ldots, v_{N-1}$, otherwise not. In this case, equation (21) can be complemented by either equation (23) or the following one:

$$
b_{N}-\sqrt{\mathcal{D}_{N}}=\frac{2 \nu_{N}}{k_{N}} \cosh k_{N} d .
$$

Of course, it is better to use the system that comprises equations (21) and (23) because the right-hand side terms in these equations are proportional.

If the measured free-surface elevation corresponding to $\nu_{N}$ cannot be represented as a linear combination of $v_{1}, \ldots, v_{N-1}$, then $\nu_{N}=\nu_{N}^{(-)}<\nu_{1}^{(+)}$, in which case the elevation is a linear combination of eigenfunctions that correspond to the eigenvalue $k_{N}^{2}$ of problem (10) the second largest after $k_{1}^{2}$. In this case, equation (21) must be complemented by (24).

Thus, we arrive at the following procedure for reducing the inverse sloshing problem to a system of two equations.

Procedure. Let $v_{1}, \ldots, v_{N-1}$ be the set of linearly independent eigenfunctions of problem (10) corresponding to $k_{1}^{2}$. If the observed elevation of the free surface that corresponds to the measured value $\nu_{N}$ has a representation as a linear combination of $v_{1}, \ldots, v_{N-1}$, then $\rho$ and $d$ must be determined from equations (21) and (23). Otherwise, equations (21) and (24) must be used.

The simplest case is when the fundamental eigenvalue of problem (10) is simple, that is, $N=2$. Then the above procedure reduces to examining whether the free surface elevations corresponding to $\nu_{1}$ and $\nu_{2}$ are proportional or not. In the case of proportionality, equations (21) and (23) must be used. Equations (21) and (24) are applicable when there is no proportionality.

\section{Solution of the transcendental systems} and $h$.

In this section, we consider the question how to solve systems (21) and (24), and (21) and (23) for finding $\rho$

\subsection{System (21) and (23)} follows:

Equations (21) and (23) can be easily simplified. Indeed, the sum and difference of these equations are as

$$
b_{1}=\frac{\nu_{N}+\nu_{1}}{k_{1}} \cosh k_{1} d \quad \text { and } \quad \mathcal{D}_{1}=\left(\frac{\nu_{N}-\nu_{1}}{k_{1}}\right)^{2} \cosh ^{2} k_{1} d .
$$


Substituting the first expression into the second equation (see formulae (18) and (19)), we obtain:

$$
(\rho-1) \sinh k_{1} h \sinh k_{1}(d-h)=\frac{\nu_{N} \nu_{1}}{k_{1}^{2}} \cosh k_{1} d
$$

whereas the first equation itself has the following form:

$$
(\rho-1) \cosh k_{1} h \sinh k_{1}(d-h)=\frac{\nu_{N}+\nu_{1}}{k_{1}} \cosh k_{1} d-\sinh k_{1} d .
$$

The last two equations immediately yield:

$$
\tanh k_{1} h=\frac{\nu_{N} \nu_{1}}{k_{1}\left(\nu_{N}+\nu_{1}-\nu_{1}^{W}\right)},
$$

where formula (11) is applied. Thus we are in a position to formulate the following.

Proposition 6. Let $\nu_{1}$ and $\nu_{N} \neq \nu_{1}$ be the smallest two sloshing eigenvalues measured for a two-layer fluid occupying $W=D \times(-d, 0)$. Let also:

$$
0<\frac{\nu_{N} \nu_{1}}{k_{1}\left(\nu_{N}+\nu_{1}-\nu_{1}^{W}\right)}<\tanh k_{1} d
$$

where $k_{1}^{2}$ is the fundamental eigenvalue of problem (10) in $D$ and $\nu_{1}^{W}$ is defined by formula (11) with $k=k_{1}$. If Procedure guarantees that $\rho$ and h satisfy equations (21) and (23), then:

$$
h=\frac{1}{k_{1}} \tanh ^{-1} \frac{\nu_{N} \nu_{1}}{k_{1}\left(\nu_{N}+\nu_{1}-\nu_{1}^{W}\right)},
$$

whereas $\rho$ is determined either by (25) or by (26) with this $h$.

We recall that $\tanh ^{-1} z=\frac{1}{2} \ln \frac{1+z}{1-z}$ (see [11], Section 4.6).

\subsection{System (21) and (24)}

Since equations (21) and (24) have the same form, we treat them simultaneously. Eliminating square roots, we get:

$$
(\rho-1) \sinh k_{j}(d-h)\left(\nu_{j} \cosh k_{j} h-k_{j} \sinh k_{j} h\right)=\frac{\nu_{j}}{k_{j}}\left(\nu_{j} \cosh k_{j} d-k_{j} \sinh k_{j} d\right), \quad j=1, N,
$$

which is linear with respect to $\rho-1$. Taking into account formula (11), we write this system in the form:

$$
(\rho-1) \sinh k_{j}(d-h)\left(k_{j} \sinh k_{j} h-\nu_{j} \cosh k_{j} h\right)=\frac{\nu_{j}}{k_{j}}\left(\nu_{j}^{W}-\nu_{j}\right) \cosh k_{j} d, \quad j=1, N,
$$

where the right-hand side term is positive in view of Corollary 1 . We eliminate $\rho-1$ from system (27), thus obtaining the following equation for $h$ :

$$
\begin{aligned}
& \frac{\nu_{1}}{k_{1}}\left(\nu_{1}^{W}-\nu_{1}\right) \cosh k_{1} d \sinh k_{N}(d-h)\left(k_{N} \sinh k_{N} h-\nu_{N} \cosh k_{N} h\right) \\
& \quad-\frac{\nu_{N}}{k_{N}}\left(\nu_{N}^{W}-\nu_{N}\right) \cosh k_{N} d \sinh k_{1}(d-h)\left(k_{1} \sinh k_{1} h-\nu_{1} \cosh k_{1} h\right)=0 .
\end{aligned}
$$

Let us denote by $U(h)$ the expression on the left-hand side and investigate its behaviour for $h \geq 0$, because solving equation (28) is equivalent to finding zeroes of $U(h)$ that belong to $(0, d)$.

It is obvious that $U(d)=0$, and we have that:

$$
U(0)=-\nu_{N} \nu_{1}\left(\frac{\nu_{1}^{W}-\nu_{1}}{k_{1}} \cosh k_{1} d \sinh k_{N} d-\frac{\nu_{N}^{W}-\nu_{N}}{k_{N}} \cosh k_{N} d \sinh k_{1} d\right) .
$$

After applying formula (11), this takes the form:

$$
U(0)=\left(\nu_{N}^{W} \nu_{1}-\nu_{N} \nu_{1}^{W}\right) \frac{\nu_{N} \nu_{1}}{k_{N} k_{1}} \cosh k_{N} d \cosh k_{1} d,
$$

and so $U(0)$ is positive, negative or zero simultaneously with $\nu_{N}^{W} \nu_{1}-\nu_{N} \nu_{1}^{W}$. 
We have that

$$
\begin{aligned}
U^{\prime}(h) & =\frac{\nu_{1} k_{N} \cosh k_{1} d}{k_{1}}\left(\nu_{1}^{W}-\nu_{1}\right)\left[k_{N} \sinh k_{N}(d-2 h)+\nu_{N} \cosh k_{N}(d-2 h)\right] \\
& -\frac{\nu_{N} k_{1} \cosh k_{N} d}{k_{N}}\left(\nu_{N}^{W}-\nu_{N}\right)\left[k_{1} \sinh k_{1}(d-2 h)+\nu_{1} \cosh k_{1}(d-2 h)\right], \\
\frac{U^{\prime \prime}(h)}{2} & =\frac{\nu_{N} k_{1}^{2} \cosh k_{N} d}{k_{N}}\left(\nu_{N}^{W}-\nu_{N}\right)\left[k_{1} \cosh k_{1}(d-2 h)+\nu_{1} \sinh k_{1}(d-2 h)\right] \\
& -\frac{\nu_{1} k_{N}^{2} \cosh k_{1} d}{k_{1}}\left(\nu_{1}^{W}-\nu_{1}\right)\left[k_{N} \cosh k_{N}(d-2 h)+\nu_{N} \sinh k_{N}(d-2 h)\right] .
\end{aligned}
$$

Then, formula (11) yields the following asymptotic formula:

$$
U(h) \sim(d-h)\left(\nu_{N}^{W}-\nu_{N}\right)\left(\nu_{1}^{W}-\nu_{1}\right)\left[\frac{\nu_{1} k_{N}}{k_{1}}-\frac{\nu_{N} k_{1}}{k_{N}}\right] \cosh k_{N} d \cosh k_{1} d \quad \text { as } d-h \rightarrow+0 .
$$

Since equation (28) is obtained under the assumption that $\nu_{N}=\nu_{N}^{(-)}$and $\nu_{1}=\nu_{1}^{(-)}$, Corollary 1 yields that each factor in the asymptotic formula is positive except for the difference in the square brackets.

The next lemma gives a condition providing a relationship between the value $U(0)$ and the behavior of $U(h)$ for $h<d$ and sufficiently close to $d$.

Lemma 2. If the following inequality holds:

$$
\frac{\nu_{1} k_{N}}{k_{1}}-\frac{\nu_{N} k_{1}}{k_{N}} \leq 0
$$

then $U(0)<0$ and $U(h)<0$ when $h<d$ and sufficiently close to $d$.

Proof. Let us prove the inequality $U(0)<0$ first. Since

$$
\nu_{N}^{W} \nu_{1}-\nu_{N} \nu_{1}^{W}=\nu_{1} k_{N} \tanh k_{N} d-\nu_{N} k_{1} \tanh k_{1} d,
$$

according to formula (11). Furthermore, it follows from (31) that:

$$
\nu_{N}^{W} \nu_{1}-\nu_{N} \nu_{1}^{W} \leq \nu_{N} k_{1}^{2} d\left[\frac{\tanh k_{N} d}{k_{N} d}-\frac{\tanh k_{1} d}{k_{1} d}\right]<0,
$$

because $z^{-1} \tanh z$ is a monotonically decreasing function on $(0,+\infty)$ and $k_{1}<k_{N}$. Then (29) implies that $U(0)<0$.

If inequality (31) is strict, then the second assertion immediately follows from the asymptotic formula (30).

In the case of equality in (31), the asymptotic formula (30) must be extended to include the second-order term with respect to $d-h$ (see the second derivative above). Thus we obtain that:

$$
\begin{aligned}
U(h) & \sim(d-h)^{2}\left\{\frac{\nu_{N} k_{1}^{2} \cosh k_{N} d}{k_{N}}\left(\nu_{N}^{W}-\nu_{N}\right)\left[k_{1} \cosh k_{1} d-\nu_{1} \sinh k_{1} d\right]\right. \\
& \left.-\frac{\nu_{1} k_{N}^{2} \cosh k_{1} d}{k_{1}}\left(\nu_{1}^{W}-\nu_{1}\right)\left[k_{N} \cosh k_{N} d-\nu_{N} \sinh k_{N} d\right]\right\} \quad \text { as } d-h \rightarrow+0 .
\end{aligned}
$$

Applying the equality $\nu_{N}=\nu_{1}\left(k_{N} / k_{1}\right)^{2}$ along with formula (11), we write the expression in braces as follows:

$$
\nu_{1} k_{N} k_{1}^{-1} \cosh k_{N} d \cosh k_{1} d\left[\left(\nu_{N}^{W}-\nu_{N}\right)\left(k_{1}^{2}-\nu_{1} \nu_{1}^{W}\right)-\left(\nu_{1}^{W}-\nu_{1}\right)\left(k_{N}^{2}-\nu_{N} \nu_{N}^{W}\right)\right],
$$

and we have in the square brackets:

$$
k_{1}^{2} \nu_{N}^{W}-k_{N}^{2} \nu_{1}^{W}+\nu_{N}^{W} \nu_{1}^{W} \nu_{N}-\nu_{N}^{W} \nu_{1}^{W} \nu_{1}+\nu_{1}^{W} \nu_{N} \nu_{1}-\nu_{N}^{W} \nu_{N} \nu_{1}
$$

Substituting $\nu_{N}=\nu_{1}\left(k_{N} / k_{1}\right)^{2}$, we see that this expression is the following quadratic polynomial in $\nu_{1}$ :

$$
\left(\nu_{1}^{W}-\nu_{N}^{W}\right)\left(k_{N} / k_{1}\right)^{2} \nu_{1}^{2}+\nu_{N}^{W} \nu_{1}^{W}\left[\left(k_{N} / k_{1}\right)^{2}-1\right] \nu_{1}+\nu_{N}^{W} k_{1}^{2}-\nu_{1}^{W} k_{N}^{2} .
$$

Its first and third coefficients are negative (for the latter one this follows from formula (32) because it is equal to the expression in the square brackets multiplied by a positive coefficient). On the other hand, the second coefficient is positive. Therefore, the last expression is negative when $\nu_{1}>0$, which implies that the right-hand side of the last asymptotic formula is negative. This completes the proof of the second assertion.

The immediate consequences of Lemma 2 are the following two corollaries. 
Corollary 4. If inequality (31) holds, then equation (28) for $h$ (and the inverse sloshing problem for a two-layer fluid occupying $W$ ) either has no solution or have more than one solution.

Proof. Inequality (31) implies that $U(0)<0$ and $U(h)<0$ for $h<d$, but sufficiently close to $d$. Hence $U(h)$ either has no zeroes on $(0, d)$, or has more than one zero.

Corollary 5. Let $\nu_{1}$ and $\nu_{N} \in\left(\nu_{1}, \nu_{N}^{W}\right)$ be the smallest two measured sloshing eigenvalues for a two-layer fluid occupying $W=D \times(-d, 0)$. Then a necessary condition that equation (28) has a unique solution $h$ is the simultaneous validity of the following two inequalities:

$$
\frac{\nu_{1} k_{N}}{k_{1}}-\frac{\nu_{N} k_{1}}{k_{N}}>0 \quad \text { and } \quad \nu_{N}^{W} \nu_{1}-\nu_{N} \nu_{1}^{W}<0
$$

Proof. Let equation (28) have a unique solution on $(0, d)$. According to Corollary 4, inequality (31) contradicts to this assumption, and so the first inequality (33) must hold. Then the asymptotic formula (30) implies that $U(h)>0$ when $h<d$ and is sufficiently close to $d$. Hence, the assumption that equation (28) has a unique solution on $(0, d)$ implies that either the second inequality (33) is true or $\nu_{N}^{W} \nu_{1}=\nu_{N} \nu_{1}^{W}$. Let us show that this equality is impossible which completes the proof.

Indeed, according to formula (29), the latter equality means that $U(0)=0$, and so

$$
U(h) \sim h\left(\nu_{N}^{W} \nu_{1}^{W}-\nu_{N} \nu_{1}\right)\left(\frac{\nu_{1} k_{N}}{k_{1}}-\frac{\nu_{N} k_{1}}{k_{N}}\right) \cosh k_{N} d \cosh k_{1} d \text { as } h \rightarrow+0 .
$$

Here, the formula for $U^{\prime}$ is used along with (11) and the fact that $\nu_{N}^{W} \nu_{1}=\nu_{N} \nu_{1}^{W}$. Since the first inequality (33) is already shown to be true, we have that $U(h)>0$ when $h \neq 0$, but is sufficiently close to +0 . Since we also have that $U(h)>0$ when $h<d$ and is sufficiently close to $d$, we arrive at a contradiction to the assumption that equation (28) has a unique solution on $(0, d)$.

Now we are in a position to formulate the following

Proposition 7. Let $\nu_{1}$ and $\nu_{N} \in\left(\nu_{1}, \nu_{N}^{W}\right)$ be the smallest two sloshing eigenvalues measured for a two-layer fluid occupying $W=D \times(-d, 0)$. If inequalities (33) hold for $\nu_{1}$ and $\nu_{N}$, then either of the following two conditions is sufficient for equation (28) to have a unique solution $h \in(0, d)$ :

(i) $U^{\prime}(h)$ vanishes only once for $h \in(0, d)$;

(ii) $U^{\prime \prime}(h)<0$ on $(0, d)$.

Proof. Inequalities (33) and formulae (29) and (30) imply that $U(0)<0$ and $U(h)>0$ for $h<d$ and sufficiently close to $d$. Then, either of the formulated conditions is sufficient to guarantee that equation (28) has a unique solution on $(0, d)$.

It is an open question whether equation (28) can have more than one solution (consequently, at least three solutions), when inequalities (33) are fulfilled.

\section{Conclusions}

We have considered the direct and inverse sloshing problems for a two-layer fluid occupying an open container. Several results obtained for the direct problem include:

(i) variational principle and its corollary concerning inequality between the fundamental sloshing eigenvalues for homogeneous and two-layer fluids occupying the same bounded domain.

(ii) Analysis of the behavior of eigenvalues for containers with vertical walls and horizontal bottoms. It demonstrates that there are two sequences of eigenvalues with the same eigenfunctions corresponding to eigenvalues having the same number in each of these sequences. The elements of these sequences are expressed in terms of eigenvalues for the Neumann Laplacian in the two-dimensional domain which is a horizontal cross-section of the container.

(iii) In the particular case of infinitely deep container with vertical boundary, eigenvalues and eigenfunctions for homogeneous and two-layer fluids are the same for any depth of the interface. This makes senseless the inverse sloshing problem in a two-layer fluid occupying such a container.

Inverse sloshing problem for a two-layer fluid, that occupies a container of finite constant depth with vertical walls, is formulated as the problem of finding the depth of the interface and the ratio of fluid densities from the smallest two eigenvalues measured by observing them at the free surface. This problem is reduced to two transcendental equations depending on the measured eigenvalues. There are two systems of such equations and to obtain these systems one has to take into account the behavior of the observed free surface elevation. Sufficient conditions for solubility of both systems have been found. 


\section{References}

[1] Fox D. W., Kuttler J. R. Sloshing frequencies. Z. angew. Math. Phys., 1983, 34, P. 668-696.

[2] Kopachevsky N.D., Krein S. G. Operator Approach to Linear Problems of Hydrodynamics. Birkhäuser, Basel-Boston-Berlin, 2001, $384 \mathrm{pp}$.

[3] Cadby J. R., Linton C. M. Three-dimensional water-wave scattering in two-layer fluids. J. Fluid Mech., 2000, 423, P. 155-173.

[4] Linton C. M., Cadby J. R. Trapped modes in a two-layer fluid. J. Fluid Mech., 2003, 481, P. $215-234$.

[5] Kuznetsov N., McIver M., McIver P. Wave interaction with two-dimensional bodies floating in a two-layer fluid: uniqueness and trapped modes. J. Fluid Mech., 2003, 490, P. 321-331.

[6] Karazeeva N. A., Solomyak M.Z. Asymptotics of the spectrum of the contact problem for elliptic equations of the second order. Selecta Math. Sovietica, 1987, 6 (1), P. 151-161.

[7] Courant R., Hilbert D. Methods of Mathematical Physics. Vol. 1. Interscience, N Y, 1953, 561 pp.

[8] Berry M. Singular limits. Physics Today, 2002, 5, P. 10-11.

[9] Bandle C. Isoperimetric Inequalities and Applications. Pitman, London, 1980, 228 pp.

[10] Lamb H. Hydrodynamics. Cambridge University Press, Cambridge, 1932, 738 pp.

[11] Abramowitz M., Stegun I. A. Handbook of Mathematical Functions. Dover, Mineola, N Y, 1965, 1046 pp. 